\title{
Haptic distal spatial perception mediated by strings: Size at a distance and egocentric localization based on ellipse geometry
}

\author{
Patrick A. Cabe \\ Published online: 14 November 2012 \\ (C) Psychonomic Society, Inc. 2012
}

\begin{abstract}
Participants explored ellipse perimeters defined by fixed-length strings, held taut by their moving finger, with ends attached to two fixed hooks (foci). Participants haptically judged ellipse interfocal distance (IFD; in effect, exocentric separation, or size at a distance) or location of ellipse major axes (i.e., egocentric localization relative to the moving finger). In Experiment 1A, perceivers made reliable and accurate multialternative forced choice IFD judgments. Experiment 1B showed similar reliability for direct estimations but showed greater scaling error. In Experiment 2, perceivers reliably localized ellipse major axes. Both experiments derived from a priori geometrical analysis, consistent with the Gibsonian perceptual research program and previous string-mediated haptic distal spatial studies. Results are discussed with respect to haptic perception as a telemodality and to dynamic touch.
\end{abstract}

Keywords Haptic perception - Spatial perception - Dynamic touch $\cdot$ Size perception

Organisms typically perceive spatial layout relative to themselves by means of the traditional telemodalities (von Fieandt, 1966), vision or audition (Wightman \& Jenison, 1995), sensory systems capable of picking up information about objects, surfaces, substances, and events distal to the perceiver's station point. Although not typically regarded as such, might haptic perception, under at least some limited conditions,

\section{P. A. Cabe $(\bowtie)$}

Department of Psychology,

University of North Carolina at Pembroke,

Pembroke, NC 28372, USA

e-mail: patrick.cabe@uncp.edu

Present Address:

P. A. Cabe

924 Fearrington Post,

Pittsboro, NC 27312, USA function as a telemodality? The possibility has rarely been contemplated and has essentially been ignored empirically.

\section{String mediation of haptic distal spatial perception: Theoretical context}

Touch and haptics pick up information about objects, surfaces, substances, and events, generally via immediate physical contact with receptor surfaces (e.g., Hayward, 2008; Lederman \& Klatzky, 1993). Recent experiments (Cabe, 2011; Cabe \& Hofman, 2012) have shown string-mediated haptic distal spatial perception to be possible, absent such immediate contact. In those studies, the perceiver's finger held taut one or more strings connecting the finger to distal spatial locations and estimated spatial properties of the resulting scenarios. Perceptual judgments depended on changing force relationships available at the finger. Finger movements were limited to either vertical or horizontal trajectories, with string tension maintained against the finger by hook-suspended weights. The present experiments diverged from those studies in using strings of fixed length, with participants pulling the string taut against fixed attachment points. Consequently, finger trajectories were constrained to planar elliptical paths. Because force directions and magnitudes could differ idiosyncratically and because vector analyses of force patterns are indeterminate (i.e., yield multiple possible solutions or no solution), physical-mechanical analyses of force relationships do not readily provide definitive hypotheses about spatial properties to be perceived. However, other elements, based on ellipse geometry, are determinate and suggest testable hypotheses that the data reported support (see the Appendix).

Visual spatial perception is perhaps the most studied area in all perceptual research. As such, visual perceptual studies (e.g., Sedgwick, 1986) suggest a wide variety of possible problems that might be instantiated in string-mediated haptic analogs. Cabe (2011) illustrated this theme in studying 
haptic looming, inspired by studies of optical looming. In that same article, Cabe (2011) also demonstrated a haptic version of Lee's $(1976,2009)$ optical tau. Cabe and Hofman (2012) employed a string-mediated haptic procedure analogous to the optical bypass geometry investigated by Kaiser and Mowafy (1993).

The present study demonstrates haptic analogs of two other phenomena central to visual spatial perception. First, perception of ellipse interfocal distance (IFD) is analogous to size at a distance (exocentric separation, or size at a distance), historically an important perceptual problem. Second, perception of ellipse major axis localization is analogous to egocentric localization - that is, where in the environment a particular feature is located, relative to a (moving) point of observation. Note that the aim of the experiments reported here was only to demonstrate these novel and surprising effects. Many questions subsidiary to these initial investigations remain to be pursued; some of those are indicated in the General Discussion section.

These studies are important for several reasons. First and most important, they explore a realm of spatial perception not widely regarded as feasible. Haptic perception of spatial properties of objects and of spatial arrangements of environmental features typically has been taken to require either immediate contact with such properties and features (Gibson, 1962; Hayward, 2008; Lederman \& Klatzky, 1993, 2009) or exploration by means of tools or prostheses, the properties of which are themselves often important to perceptual performance (e.g., mass, moment of inertia, and vibratory characteristics). The latter point is discussed at length in Burton (1993), Cabe (2011), and Hayward (2008), although one example of rods as mediating devices not relying on their physical properties exists in the studies of bimanual triangulation of distance by Cabe, Wright and Wright (2003).

String-mediated perception, on the other hand, may occur in the absence of immediate contact between effectors and the properties to be perceived. Neither must such perception depend on intervening substantial properties of the string (e.g., mass). Rather, the important element of stringmediated distal haptic perception is resistance to tension in low-mass, small-dimensioned, flexible cordage (Cabe, 2011; Cabe \& Hofman, 2012). The only other apparent empirical essay into this general sphere is the work of Kinsella-Shaw and Turvey (1992), who found that participants could readily judge distances to a mass suspended on a taut vibrating cord.

Second, Cabe's (2011; Cabe \& Hofman, 2012) studies grew out of a priori physical-mathematical analyses, which revealed that haptic distal spatial perception might be possible. Empirical results verified that perceivers could, in fact, use the potential information available. Cabe's theoretical framework was that proposed by James Gibson (1966, 1979), which at the very least serves as a convenient entry to this and other perceptual research. Gibson asserted that perception utilizes structure in stimulus arrays, including variables that transform as events unfold in the environment and as organisms explore it. Transforming arrays, per Gibson, contain invariant structure specific to environmental objects, surfaces, substances, and events. Sedgwick (1973; see also Turvey, Solomon, \& Burton, 1989) outlined a cognate research program, emphasizing that the crucial first step is to analyze stimulus arrays to discover potentially informative patterns of energy flux. The next step, then, is to test appropriately sampled organisms to see whether they can exhibit the putative perceptual capability suggested by the array analysis.

Cabe's (2011; Cabe \& Hofman, 2012) work is entirely consistent with this pattern and, by extension, therefore supportive of Gibson's $(1966,1979)$ perceptual theory, albeit far from a crucial test. Furthermore, Cabe proposed a strategy for identifying available stimulus array information: If a physical-mathematical expression that describes some object, surface property, substance, or event can be connected (e.g., via an equation) to a physical-mathematical expression that describes properties of a stimulus array, then one can claim that information exists in the stimulus array for the environmental properties in question. The physical-mechanical analyses displayed in Cabe (2011), in Cabe and Hofman (2012), and in the Appendix here illustrate that pattern with regard to string-mediated distal spatial perception. Note that such array analysis may suggest some form of available informative structure, without obviating the possibility that other analyses may exist (cf. Cutting, 1986) that might point to alternative patterns of array information. Stimulus array structure proposes potential perceptual capability. Discovering that the hypothesized perceptual ability exists, although suggestive, does not necessarily imply that the array properties that yielded the hypothesis in fact informed perceptual performance. The specific informational basis for a given performance requires both additional fine-grained empirical study and, possibly, additional forms of array analysis.

This general strategy applies even in instances in which such perception may never have been seen in the ongoing behavior of the organism. That is, potential stimulus array information can exist that a given population of perceivers has never exploited, even though perceptual systems, if called upon to do so, might be capable of utilizing the information available (i.e., the perceptual capability may be latent).

Third, string-mediated haptic perception extends the scope of dynamic touch perception in a new direction. The physical-mechanical components defining dynamic touch (Amazeen, 1999; Gibson, 1966) are forces generated by exploring effectors and reactive forces sensible by receptors in the skin, joints, and muscles. Much of the extant dynamic touch research has focused on wielded objects (Carello \& 
Turvey, 2004; Turvey, 1996; Turvey \& Carello, 1995). Results from such studies clearly show that humans can perceive object length, shape, and orientation, among other spatial properties. Relevant to the present work, Garrett, Barac-Cikoja, Carello, and Turvey (1996) have studied haptic perception of size at a distance, using handheld rods.

The primary candidates for informative structure in stimulus arrays supporting string-mediated haptic distal spatial perception are (a) force relationships and changes in force sensible by the haptic system, (b) angular relationships and changes in angles between or among string segments around the finger or other effector, and (c) effector position and changes in effector position. All of these may be present simultaneously. Those variables also have properties characteristic of dynamic touch.

Both Cabe (2011) and Cabe and Hofman (2012) focused primarily on changing force relationships. The results reported by Cabe (2011) converged on the conclusion that participants judged impending finger-hook collinearity in haptic looming by use of changing force relationships. Cabe (2011) acknowledged another possibility: Participants could have used the change in angle between string segments as the finger changed position. As the finger approached collinearity with the hooks, the angle between the string segments increased, while the contact with the finger (termed the wrap angle) progressively decreased, approaching zero at collinearity. Cabe (2011) attempted to control for the possible influence of wrap angle by using manipulanda (a rod in contact with the string or a rigid ring attached to the string, the latter technique also employed by Cabe \& Hofman, 2012), with force changes and finger position changes still available. Performance in the absence of wrap angle information did not differ from that with wrap angle available. None of Cabe's (2011) procedures, however, tested changes in wrap angle per se, either alone or in combination with finger position changes. Although the spatial perceptual focus is different from that in Cabe (2011), in the present experiments, wrap angle and finger position provide the most likely information for the perceptual outcomes tested.

In earlier investigations, string tension depended on small weights suspended at the free ends of strings, countered by muscular action. Consequently, information for spatial properties mediated by the strings resided in the resulting force relationships. Those relationships, as Cabe (2011; Cabe \& Hofman, 2012) outlined, change as the finger moves; force variations implied distal spatial relationships.

Here, I show that perception of two important spatial properties - separation of two distal points (exocentric separation, or size at a distance) and egocentric localization of the line through those points - occurs when an exploring finger holds taut a fixed-length string secured to two distal points. Those conditions confine the finger's movement to an elliptical trajectory. Exocentric separation of two points is operationalized as the distance between the two foci of the ellipses presented (IFD); egocentric localization is of the major axis of the ellipse, the line through the two foci. In contrast to procedures used in earlier studies (Cabe, 2011; Cabe \& Hofman, 2012), tension in the string is entirely the product of muscular exertion applied by participants, rather than suspended weights. The participant alone controls finger force magnitudes and directions, in indeterminate ways. Consequently, changes in wrap angle at the finger and in effector position constitute the most likely potential information for perception of the spatial properties in question.

As is described in the Appendix, the hypotheses tested in the present experiments derive from ellipse geometry. Ellipses have featured in both visual and haptic perception studies. Mostly, however, the concern in such research has been on the perimeter or surface of the ellipses used. For example, Aleci, Piana, and Anselmino (2010; see also Dresp, Silvestri, \& Motro, 2007; Van der Horst \& Kappers, 2008) used ellipses with varying curvature to show that humans demonstrate a visual hyperacuity for curvature (the ratio of major to minor axes). While, in principle, all the properties of a given ellipse are implied by the perimeter, apparently no studies have been published on perception of other spatial properties related to the ellipse.

\section{Scope of the present experiments}

Two main experiments are described below. In the first experiment (with two variations), participants attempted to locate IFDs - in effect, estimating the distances between the foci of ellipses of several sizes and shapes. In Experiment 2, participants judged the location of the major axes (lines through ellipse foci) of several ellipses, relative to a moving finger, within a fixed frame. Both experiments tested hypotheses generated on the basis of a priori physical-mechanical and geometrical analysis (shown in the Appendix). Again, the present experiments demonstrate that the targeted effects do occur, although a variety of research questions about them remain open.

\section{Experiment 1A}

Experiment 1A tested the proposition that perceivers constrained to move their fingers along an elliptical path, defined by a string held taut by the finger, could judge the location of the two ends of the fixed-length string attached to the ellipse foci, thereby implicitly estimating the ellipse IFD. Both ellipse IFD and shape (i.e., the ratio of minor to major axis length) varied. Because the task was quite unusual and, therefore, likely to be difficult, I simplified it to a 
multialternative forced choice procedure. That is, participants explored the perimeters of the ellipses imposed by the apparatus and were required to choose which of a set of specified alternatives represented the actual ellipse IFD. Multiple alternative forced choice tasks are relatively common in the literature-for example, Burton, Turvey, and Solomon (1990) - in testing perception of object shape by wielding.

Two main hypotheses were tested. First, if haptic information for IFD exists under the conditions tested, judged IFD should correlate relatively strongly with actual IFD. Second, if (per the analysis in the Appendix) the information resides in changing angles between the string segments, in kinesthetic sensitivity to hand-arm movement or both, judged IFD should be relatively strongly correlated with those informational variables. Furthermore, the analysis in the Appendix suggests that string length per se should be relatively unimportant for IFD judgments.

\section{Method}

\section{Participants}

Twenty members of the University of North Carolina at Pembroke community participated (18-47 years of age; median, 19; 10 male, 10 female; all right-handed). Most were students in psychology classes; some received course credit for participation. All were naive with respect to the experimental hypotheses and methods. None reported any neurological or motoric difficulties.

\section{Apparatus}

A vertically oriented, free-standing wood frame, approximately $155 \mathrm{~cm}$ high $\times 157 \mathrm{~cm}$ wide, constructed of nominal 6-in. lumber, supported five pairs of hooks. The inside width of the frame was $150 \mathrm{~cm}$; the transverse board to which the hooks were screwed was $115 \mathrm{~cm}$ above the floor. Hooks were located $25,30,35,40$, and $45 \mathrm{~cm}$ apart horizontally, centered in the frame. Any of three strings $(55,65$, and $75 \mathrm{~cm}$ long) could be attached to the hooks by means of loops at each string end. Informal preliminary testing showed these values to be adequate to demonstrate the effects hypothesized. IFD and string length combinations defined 15 ellipses, with minor-to-major axis length ratios varying from .57 (a quite elongated ellipse) to .94 (a nearly circular ellipse) and with maximum angles between the string segments varying from about $54^{\circ}$ to about $110^{\circ}$. Strings were smooth braided nylon, approximately $1.5 \mathrm{~mm}$ in diameter. A cardboard screen was attached to the front of the frame, projecting toward the participant at an angle; the screen occluded hooks, strings, and the participant's hand and forearm. Figure 1 schematically depicts the geometry of the experimental setup.

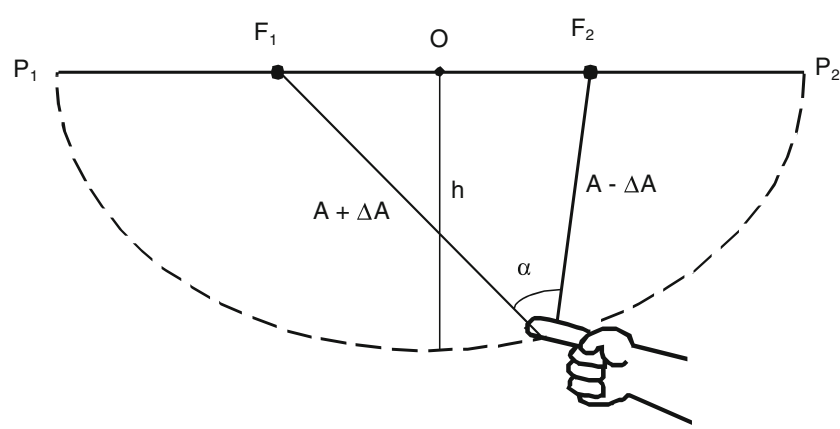

Fig. 1 Schematic diagram of finger movements in all experiments. The distance between $F_{1}$ and $F_{2}$, the ellipse foci, is the interfocal distance. $\mathrm{O}$ is the center of the ellipse major axis, the line $\mathrm{P}_{1} \mathrm{P}_{2}$, with $\mathrm{h}$ the length of the ellipse semi-minor axis. A finger holds taut a string of length $2 \mathrm{~A}$, attached at $\mathrm{F}_{1}$ and $\mathrm{F}_{2} ; \alpha$ is the angle between the string segments. As the finger moves, it traces the elliptical path shown by the dashed curve. The distance along the curve from the intersection between the curve and the semi-minor axis is $\Delta \mathrm{A}$

Participants sat in a standard armless straight chair, centered in front of the apparatus frame; the experimenter sat behind the apparatus. Participants' arms extended at an angle at the elbow (roughly $100^{\circ}$ ) that allowed comfortable movement of the extended forefinger, at a distance slightly greater than the length of the forearm (roughly $45-50 \mathrm{~cm}$ ). Little or no proximal-distal arm movement was observed during exploration; hand-finger movements were essentially in a vertical plane.

Labels on the front of the screen indicated possible hook positions (numbered 1 to 7). Numbers 2 through 6 corresponded to actual hook positions. Numbers 1 and 7 were 20 and $50 \mathrm{~cm}$ apart, respectively (outside the actual range of the hooks), to reduce central tendency errors. Labels were at approximately the participant's eye level, at a distance of approximately $50 \mathrm{~cm}$.

\section{Procedure}

After giving informed consent, participants were instructed to hold the string taut with their right forefinger, while moving the finger-hand-arm side to side laterally, essentially in a frontal-parallel plane. The movement traversed an arc that varied from about $20^{\circ}$ to about $50^{\circ}$ (depending on the particular IFD and string length combination). The participants then were to say aloud the number they thought corresponded to the hook position. Finger traverses were in essentially a vertical plane defined by the hooks and the taut strings. Trials had no time limit but usually lasted only a few seconds each. Each experimental session consisted of 90 trials in six blocks of 15 (all combinations of 3 string lengths $\times 5$ hook separations, randomized within blocks) and lasted approximately $45 \mathrm{~min}$. Although each participant experienced the same sequence of conditions, any potential order effects were 
minimized, because, with randomization within blocks, no condition could occur more than twice in succession.

Results

\section{ANOVA}

IFD estimates, calculated from the hook positions participants reported, were averaged over trials for each participant. ${ }^{1}$ Those IFD values were entered into a 5 (IFD) $\times 3$ (string length) ANOVA, with repeated measures over both factors. The only significant main effect was for IFD, $F(4$, 76) $=89.20, p<.0001, \eta^{2}=.82$. The main effect for string length was not significant, $F(2,38)=2.29, p=.12$, nor was the interaction, $F(8,152)=1.79, p=.08$. Bonferronicorrected paired $t$-tests on mean IFD estimates showed all differences to be significant, $t(19)=6.18-10.57$, maximum $p=.0001$.

\section{IFD regressions}

Multiple regressions of estimated IFD on actual IFD and string length were calculated both for the entire sample and for individual participants. Using IFD estimates averaged over participants, but not trials, ${ }^{2}$ the multiple correlation ( $n=90$ data points) for the entire group was .96 . Regression coefficients for both actual IFD and string length differed significantly from zero, in contrast to the global ANOVA finding. String length therefore appears to have affected at least the scaling of IFD judgments, perhaps via string segment angles resulting from particular combinations of IFD and string length. The regression using estimates averaged over both participants and trials ( $n=15$ stimulus values) yielded a multiple correlation of .994 (Fig. 2), again with both predictor coefficients significantly different from zero.

Multiple regressions for individuals, using data from all trials ( $n=90$ data points), gave multiple $R$ magnitudes ranging from .61 to .88 (median, .772; all $p$ s $<.0001$ ). For

\footnotetext{
${ }^{1}$ In a preliminary examination of trial-by-trial performance, I ran the ANOVA with blocks included as a variable. Initially, the blocks main effect was significant. Pairwise Bonferroni-corrected block mean comparisons showed only one pair significant, that between block 1 and block 2 . All others comparisons were nonsignificant. Omitting the first trial block (in essence, a familiarization block, in which participants first experience the range of values being tested) eliminated the significant main effect for blocks.

${ }^{2}$ What measures to use in regressions such as this is something of an issue. Least-squares regression assumes independence of observations, which cannot be assured in repeated measures designs (see Cabe, 2010, Footnote 1, for related commentary). The averaging procedure used here has many precedents in the dynamic touch literature. Furthermore, the combination of group and individual regressions gives a fair and reasonable representation of perceptual performance.
}

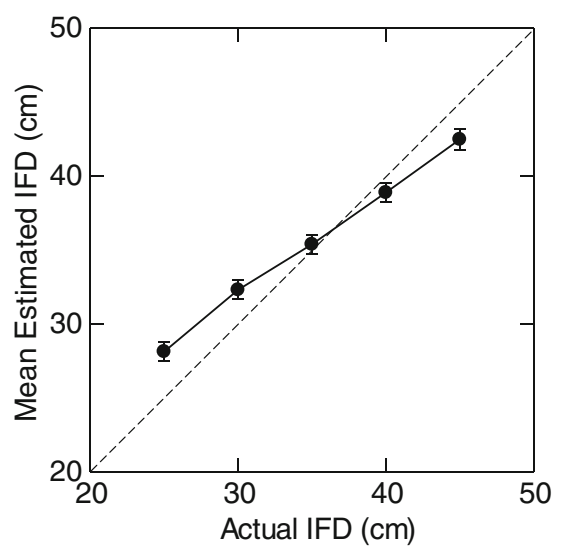

Fig. 2 Mean estimated interfocal distance (IFD) as a function of actual IFD in Experiment 1A. Estimates were not significantly different for the three string lengths used. Error bars are $\pm 1 \operatorname{SEM}(n=20$ participants per data point). The dashed line indicates ideal performance

estimates averaged over trials ( $n=15$ stimulus values), multiple $R$ magnitudes ranged from .87 to .98 (median, .956). For the latter regressions, regression coefficient magnitudes for actual IFD were all significantly different from zero (all $p \mathrm{~s}<.0001$ ). However, the coefficient for one participant was negative. Coefficients for string length differed significantly from zero in 10 of 20 cases; those coefficients varied from -0.78 to 0.72 (median, -0.29 ; maximum $p=.04$ ). Eight of the 10 significant string length coefficients were negative; the single participant who showed a negative coefficient for actual IFD also showed the largest positive coefficient for string length. However, overall there was no significant relationship between the coefficients for IFD and string length $(r=-.42, p=.06)$. One value was an outlier; removing that outlier decreased the correlation magnitude to $.25(p=.30)$.

\section{Informational variable regressions}

The analysis in the Appendix suggested that relevant stimulus array variables were (changing) angle between strings and (changing) finger position. If IFD estimates depended on use of those variables as the finger explored the ellipse perimeter, then angle and position variables should predict IFD estimates. Accordingly, for the varying IFD $\times$ string length conditions, I calculated (a) the maximum angle between the string segments (at the center point between the two hooks), (b) the angle at the point immediately below one of the hooks, as an estimate of a reasonable end point for exploratory arcs, (c) the difference between those two angles (an estimate of the angular range sampled as the finger moved), (d) straight-line distance along the ellipse perimeter between those two points (an estimate of the change in finger positions sampled), and (e) the ratio between the angular range and finger travel. Those values were, in turn, used as possible predictors of IFD judgments. 
For the group as a whole, with judgments averaged over participants but not trials ( $n=90$ data points), a stepwise regression yielded a correlation of .96. Predictors selected were angular range, finger travel, and the ratio of angular change to finger travel (maximum $p=.04$ for regression coefficients). Note that these predictors are all potentially available to the haptic system, via cutaneous and kinesthetic inputs. For measures averaged over both participants and trials $(n=15)$, the multiple correlation was .996 , with the same predictors selected.

For individuals, stepwise multiple regression yielded correlations that ranged from .62 to .95 (median, .77), all strongly significant ( $p<.0001, n=90$ data points). Patterns of predictors varied: 11 of 20 involved both angle and finger travel variables, six involved only finger travel, and three involved only angular variables. Note that these factors all depend on string length, providing a possible explanation for the variable significant regression coefficients for string length that were obtained.

\section{Discussion}

As a whole, the results indicated that participants could readily differentiate hook positions and, thus, implicitly IFDs, within the range tested, over varying ellipse geometry. That is, participants successfully perceived string-mediated size at a distance, operationalized as IFD. Globally, IFD estimates did not differ as a function of string length, within the range of lengths used. The first-order correlation between string length and IFD estimates was only -.17 , as compared with .94 for actual IFD (both $n=90$ data points). However, a number of individual participants' regression results showed a significantly nonzero coefficient for string length. Thus, the influence of string length was variable across individuals, probably because both the maximum string angle at the finger and the range of string angles explored varied with string length. Both those variables showed strong first-order associations with IFD estimates ( $r=-.85$ and .88 , respectively). As the physical-mathematical analysis in the Appendix indicates, changes in the angle at the finger (a function of the ellipse arc length explored) can inform IFD judgments. The results obtained are consistent with that analysis.

Drewing and colleagues (Drewing \& Ernst, 2006; Drewing \& Kaim, 2009; Kaim \& Drewing, 2010) proposed that a maximum likelihood estimation combination of cues explains haptic perception of shape properties. Conceivably, some similar combination of influences affected IFD judgments under the conditions tested in the present experiments. The global lack of IFD differences as a function of string length suggests that the arc length explored was adequate for the judgments made, but it would be useful to examine that variable more explicitly in future research. In the same vein, multiple regressions using physical variables (e.g., string angles, finger travel) suggested that individuals tapped different combinations of the physical-mechanical variables available. This is also consistent with recent perspectives (e.g., Jacobs \& Michaels, 2007) that, with experience, individuals explore stimulus arrays to discover more efficacious patterns of variables in order to carry out perceptual tasks.

The present results add to emerging empirical findings indicating that haptics, given a string medium, can support perception of some distal spatial properties (i.e., can function as a telemodality; von Fieandt, 1966). Cabe (2011; Cabe \& Hofman, 2012) showed that string-mediated distal haptic perception can be both quite reliable and reasonably accurate. The present results demonstrate similar qualities. The task employed in Experiment 1A, furthermore, adds to the extant literature on dynamic touch, albeit with a task notably different from the majority of those studies.

The logic that led to Experiment 1A derived from the Gibsonian perceptual research program (Sedgwick, 1973; Turvey et al., 1989), which proposed starting such research with an a priori stimulus array analysis to discover potentially informative array structure. The Appendix illustrates just that kind of analysis, yielding the hypothesis that an unusual perceptual effect might be found, a hypothesis that the data strongly supported. As was noted in the introduction, the analysis presented may represent only one pattern of informative array properties; others may exist. Whether the particular pattern of array properties available in Experiment $1 \mathrm{~A}$ is unique to the perceptual judgment tested is an open question. Cabe (2010), for example, showed that a subset of physical-mechanical information was sufficient to support length judgments of cylinders rolled by hand, where previous analyses had suggested that additional information was required.

Individual differences are a persistent issue in haptic perception, under a wide variety of test conditions. The single participant who showed a negative correlation between actual and estimated IFD evidently perceived the differences present but applied a reversed scaling relationship. Similar outcomes, particularly with novel perceptual tasks, have been reported elsewhere (Cabe, 2010, 2011; Cabe \& Hofman, 2012; Withagen \& Caljouw, 2011), all notably in haptic perceptual studies. In all those instances, participants apparently were sensitive to relevant information but applied an inverse scaling relationship (see also the discussion of similar outcomes in Experiment 1B). The range and form of individual differences found in haptic perceptual research, both the present experiments and many others in the literature, stand as a cautionary note to the interpretation of results and deserve more focused attention in future investigations. 


\section{Experiment 1B}

Experiment $1 \mathrm{~A}$ showed that participants reliably and accurately differentiated ellipse IFDs using haptic stringmediated information. The unusual nature of the original hypothesis prompted a simplified multialternative forced choice test. Because labels showed actual hook positions, participants had magnitude scaling information, so Experiment 1B focused on the narrow issue of participants' ability to scale perceived IFD. The range of IFDs and string lengths was also enlarged somewhat, to assess a broader scope of this surprising perceptual capability, although the values used overlapped those in Experiment 1A. Because the task was novel and the procedure provided no inherent scaling information, I anticipated that participants would be able to judge IFDs but that scalar accuracy should be less than in Experiment 1A. Also as in Experiment 1A, IFD judgments should be correlated with available stimulus array variables.

\section{Method}

\section{Participants}

Participants were five volunteers from the same population as in Experiment 1A (three male, two female; 18 to 24 years of age; median age, 19; one left-handed). All were naive with respect to the hypotheses of the experiment and methods used; none reported any neurological or motoric difficulties. The strong effect size for IFD estimates in Experiment 1A, as well as the limited focus for Experiment 1B, justified the use of a small sample.

\section{Apparatus}

An apparatus similar to that used in Experiment 1A was used for Experiment 1B. Actual IFDs were 20, 30, 40, 50, and $60 \mathrm{~cm}$; string lengths were 70,80 , and $90 \mathrm{~cm}$. Those values overlap those from Experiment 1A. Ratios of minor to major axis lengths varied from .52 to .97 . Hooks and strings, as well as the hand and arm, were again screened from the participant's view. The apparatus also included two vertical bars, centered in the apparatus frame and $65 \mathrm{~cm}$ apart, limiting the arc length explored to values between about $12^{\circ}$ and about $60^{\circ}$ and guaranteeing that lateral hand movements never reached the vertical level of the hooks, although allowing horizontal finger traverses greater than any IFD.

Participants made IFD estimates via a rope and pulley system, adjusting the separation of marks on two side-byside, horizontally aligned, counter-moving small-diameter ropes to equal the IFD (similar to the method used by Cabe, 2011). The portion of the ropes visible to participants and movements of the markers paralleled the ellipse major axis, at the same level above the table top as that axis, approximately at eye level. Participants adjusted the marker position by moving an approximately $40-\mathrm{cm}$ section of the rope, placed around pulleys such that the rope was parallel to and about $70 \mathrm{~cm}$ above the floor. A marker on the back of the apparatus, visible only to the experimenter, indicated estimated IFD against a millimeter-scaled ruler.

\section{Procedure}

The procedure from Experiment 1A was followed. Each session consisted of 60 trials (four trial blocks, each block containing all 15 IFD $\times$ string length combinations, in the same random order for all participants). Markers indicating IFD estimates started at the center of its traverse on half the trials and at the extremes on half the trials. Trials had no time limits, but typically each took only a few seconds. Sessions took approximately $45 \mathrm{~min}$. All participants readily completed the task.

\section{Results and discussion}

Scaling of IFD estimates was tested by means of multiple regressions on estimates averaged over participants but not trials. Using actual IFD and string length as predictors yielded a group multiple correlation ( $n=60$ data points) of $.66, p<.0001$, indicating that IFD estimates paralleled actual IFDs. The correlation, while strongly significant, was somewhat lower than that obtained in Experiment 1A. The group regression coefficient for actual IFD, but not for string length, differed significantly from zero. Coefficients for IFD were smaller than in Experiment 1A, denoting less accurate scaling. For estimates averaged over both trials and participants ( $n=15$ stimulus values), the multiple $R$ was .93 (Fig. 3), quite consistent with that found in Experiment 1A.

Multiple correlation magnitudes for IFD estimates over all trials ( $n=60$ data points) for individuals ranged in magnitude from .56 to .93 (median, .693), all $p$ s $<.0001$. These values completely overlap those found in Experiment 1A. Two participants showed negative coefficients for IFD, indicating an inverse scaling relation. Regression coefficients for actual IFD were strongly different from zero in all cases. Coefficients for string length were significantly different from zero in two of five cases, those being the 2 participants who showed the negative coefficients for actual IFD.

A stepwise regression on group average IFD estimates ( $n=60$ data points) selected predictors of (a) maximum angle and (b) the ratio of angle change to finger travel distance, while not selecting angle range or finger travel distance. The resulting correlation was .68 $(p<.0001)$. This finding is also consistent with the results of Experiment 1A. 


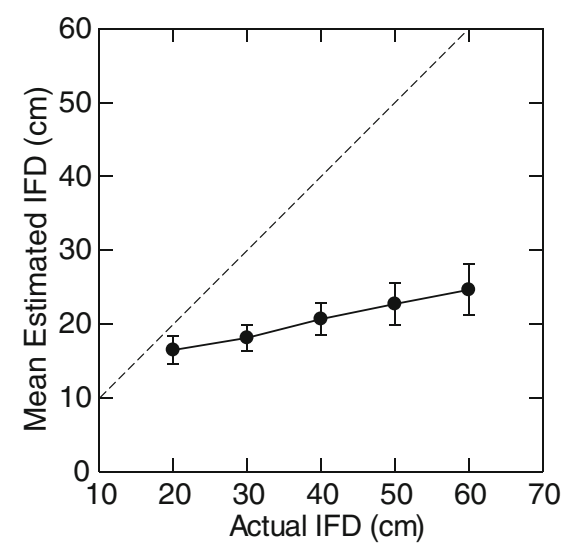

Fig. 3 Mean estimated interfocal distance (IFD) as a function of actual IFD in Experiment 1B. Estimates for the three string lengths used were not significantly different. Error bars are $\pm 1 \operatorname{SEM}(n=5$ participants per data point). The dashed line indicates ideal performance. See the Results section for Experiment 1B for explanatory details

Because participants showed both positive and negative correlations between actual and estimated IFDs and because degrees of freedom were small for pairwise $t$-tests, none of the group mean IFD differences reached significance. However, within individuals, using all IFD estimates, pairwise $t$-tests showed significant differences for between two and eight of the 10 possible comparisons, mostly for more disparate actual IFD values (i.e., all participants demonstrated the ability to differentiate IFDs).

These outcomes compare favorably with those from Experiment 1A. Note that the open-ended response procedure in Experiment 1B did not provide scaling information, as Experiment $1 \mathrm{~A}$ had, so both greater variability and scaling differences are not surprising. What is surprising is that even a small sample of participants reliably differentiated and scaled the IFD values presented, in a highly novel task. Furthermore, while substantial estimate errors occurred (more so at the higher IFD values than at the lower ones), mean estimates were reasonably within the range of actual values presented. These outcomes, then, show the pattern of definite scaling identified by Carello, Fitzpatrick, Flascher, and Turvey (1998).

Individual differences in scaling also are not surprising, given both the novelty of the task and the existence of similarly broad individual differences in other studies of dynamic touch, including instances of inverse scaling. Accurate scaling of perceptual judgments, as a rule, is a function of experience with the range of values to be estimated and feedback about such estimates, relative to some external standard (e.g., Cabe \& Wagman, 2010); participants in the present study received no such feedback. Clearly, future research might usefully examine the effects of feedback in a perceptual-learning manipulation with such IFD judgments, much as Cabe and Hofman (2012) did with haptic bypass distance judgments.
One might suggest that a possible explanation for the judgments made in both Experiments $1 \mathrm{~A}$ and $1 \mathrm{~B}$ is that participants simply judged when one of the string segments was vertical, indicating that the finger was directly below one of the hooks. The existence of negative correlations between actual and estimated IFDs argues against that explanation, because participants would have been highly unlikely to have made such judgments on the basis of their own finger positions.

\section{Experiment 2}

The Appendix suggests that haptic array information exists for localizing the major axes of ellipses traced by a moving finger. That is, given the opportunity to explore elliptical trajectories defined by specific IFDs and string lengths, perceivers should be able to judge the relative locations of varying ellipse axis locations, within a specified spatial frame. Experiment 2 tested the hypothesis that participants could make such judgments. Accordingly, conditions were arranged such that the horizontal position of the ellipse major axis varied vertically within the frame of the apparatus. Participants attempted scalar estimates of the vertical location of the horizontal major axis.

As in Experiment 1A, two main predictions emerge from the analysis in the Appendix. First, if haptic information for horizontal axis localization exists in the string-mediated scenario presented, there should be a relatively strong correlation between actual and estimated major axis positions. Second, if the information identified in that analysis is used, there should be relatively strong correlations between those informational variables and estimated horizontal axis location.

\section{Method}

\section{Participants}

Participants were 11 volunteers from the same population as in Experiments 1A and 1B (17-24 years of age; median, 18; five men, six women; nine right-handed). All were naive with respect to the experimental hypotheses and methods. None reported any neurological or motoric limitations.

\section{Apparatus}

The frame of the apparatus used in Experiment 1B was used, with wood strips attached to the inside of the frame to support horizontal boards (nominally $3 / 4 \mathrm{in}$. thick $\times 31 / 2$ in. wide $\times 60$ in. long; $1.9 \times 8.9 \times 150 \mathrm{~cm})$ at major axis heights of $150,160,170,180$, and $190 \mathrm{~cm}$ above the floor. Strings ran through holes drilled in horizontal 
support boards, with knotted ends, all with a standard $30-\mathrm{cm}$ separation (IFD), centered within the frame. The $30-\mathrm{cm}$ value was chosen as reasonably representative of the IFDs used in the previous two experiments. Because there was a possibility that starting height above the table top might act as a cue for axis height, string lengths $(50.0,67.1,85.4,104.4$, and $123.7 \mathrm{~cm})^{3}$ were chosen such that all center points of the taut strings were the same, $130 \mathrm{~cm}$ above the floor for all axis heights. These IFD and string length values also span and slightly extend those used in Experiments $1 \mathrm{~A}$ and 1B. The string lengths used resulted in ellipses having minor-to-major axis ratios between .80 and .97 . Maximum angles between string segments ranged from about $28^{\circ}$ to about $74^{\circ}$. The boards supporting the strings were clamped solidly to the framework for each trial. A wooden bar was attached to the apparatus framework $135 \mathrm{~cm}$ above the floor, but below all the heights of the boards to which the strings were attached, preventing participants from moving their fingers high enough to actually touch the horizontal string support board.

Participants adjusted a vertically oriented rope and pulley system to indicate the axis location, similar to procedures used in Experiment 1B and in other dynamic touch investigations (e.g., Cabe, 2010; Chan \& Turvey, 1991; KinsellaShaw \& Turvey, 1992). A mark on the rope, visible to participants along the left side of the apparatus frame, could be adjusted to indicate participants' judgments of axis locations. Maximum excursions for the marker were near the top of the apparatus (approximately $150 \mathrm{~cm}$ above the floor) and at the pulley at approximately the position of the participant's elbow (about $70 \mathrm{~cm}$ above the floor). Participants moved the rope upward and downward using the left hand. Participants' movements of the rope also produced movements of a calibrated mark, visible only to the experimenter at the rear side of the apparatus, to show estimated axis locations along a millimeter-scaled ruler on the back of the apparatus. As in Experiments 1A and 1B, the participant's right hand and forearm, as well as the support boards and strings (the operational elements of the apparatus), were occluded from the participant's view.

\section{Procedure}

As in Experiments 1A and 1B, participants were seated comfortably in front of the apparatus's occluding screen; again, the experimenter sat behind the apparatus. Participants used their right forefingers to hold strings taut, beginning at the center of the apparatus and then moving the finger laterally left and right, essentially in a vertical plane, while the left hand

\footnotetext{
${ }^{3}$ Note that string lengths were completely correlated with major axis heights. However, varying string length would have introduced another confound (i.e., that height at the starting point was correlated with major axis height).
}

adjusted the indicator mark on the rope to a height above the table top judged to be equivalent to the horizontal location of the ellipse major axis. Because the horizontal bar above the table top prevented the participant's finger from ever reaching horizontal, the horizontal ellipse axis locations always had to be estimated from distal information. Each session contained 50 trials (10 blocks of five axis heights, randomized within blocks in the same order for all participants). Trials had no time limit; typically, each took only a few seconds. Each session lasted about $45 \mathrm{~min}$.

Results and discussion

\section{ANOVA}

Axis localization estimates (in millimeters, per the scale on the back of the apparatus), averaged over trial blocks for each individual, were entered into a one-way repeated measures ANOVA. The effect for actual axis position was strongly significant, $F(4,40)=19.77, p<.0001, \eta^{2}=.664$. Bonferroni-corrected $t$-tests on mean differences were significant $(p<.05)$ in 8 of 10 comparisons, $t(10)=3.65-5.22$, maximum $p=.04$. The mean differences between heights of 55 and $65 \mathrm{~cm}$ and between 65 and $75 \mathrm{~cm}$ were not significant with correction but were significant without it.

\section{IFD regressions}

The correlation between estimated and actual axis heights for height estimates averaged over participants but not trials ( $n=50$ data points) was .94 . With estimates averaged over both trials and participants ( $n=5$ stimulus values), the correlation was .99 (Fig. 4). The regression slope was .36, with an intercept of $37.1 \mathrm{~cm}$.

Correlations for individual participants using all estimates $(n=50$ data points) ranged from .05 to .90 (median, .72; 9 of 11 significant, maximum $p=.0001$ ). With

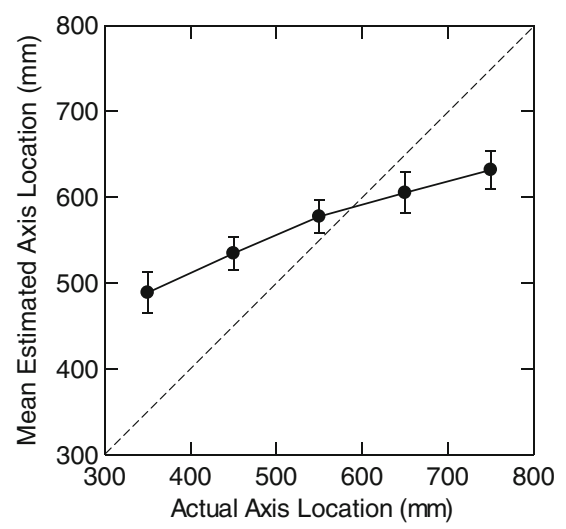

Fig. 4 Mean estimated major axis localization as a function of actual location. Error bars are $\pm 1 \operatorname{SEM}(n=11$ participants per data point). The dashed line indicates ideal performance 
estimates averaged over trials, the median correlation $(n=5$ stimulus values) was .97 (range, .33-.98; 9 of 11 significant, maximum $p=.018$ ).

\section{Informational variable regressions}

If major axis localization estimates depended on use of the (changing) angle between strings as the finger explored the ellipse perimeter, then angle and position variables should predict those estimates. Accordingly, for the varying axis height conditions, I calculated (a) the maximum angle between the string segments (at the center point between the two hooks), (b) the angle at the point immediately below one of the hooks, (c) the difference between those two angles (an estimate of the angular range sampled as the finger moved), (d) straight-line distance along the ellipse perimeter between those two points (an estimate of the change in finger positions sampled), and (e) the ratio between the angular range and finger travel. Those values were, in turn, used as possible predictors of major axis localization judgments.

For the group as a whole, with judgments averaged over participants but not trials ( $n=50$ data points), a stepwise regression yielded a correlation of .95. Predictors selected were angular range and the ratio of angular change to finger travel (maximum $p=.0013$ for regression coefficients). Note that these predictors are both potentially available to the haptic system, via cutaneous and kinesthetic inputs.

For individuals, stepwise multiple regression yielded correlations that ranged from zero to .92 (median, .72), 9 of 11 strongly significant ( $p<.0002, n=50$ data points). The two participants who showed nonsignificant correlations with array variables also showed nonsignificant correlations between actual and estimated major axis locations. Patterns of predictors varied: Seven of the nine significant correlations involved both angle and finger travel variables, while one each involved only finger travel or only angular variables. Those patterns are consistent with those seen for IFD estimates.

Clearly, participants reliably localized major axis positions within the geometry of ellipses defined by fixed foci and fixed-length strings, within the frame established by the apparatus. String lengths varied with axis location, a constraint required to equate finger starting positions for the axes tested; therefore, this experiment leaves ambiguous whether the ability to estimate major axis location was independent of string length, as the analysis in the Appendix suggests would be true. It is unclear how one might unconfound axis location and string length, without introducing a different potential confound with finger starting position.

The slope of the regression line less than unity and the intercept greater than zero indicate that localization scaling was somewhat inaccurate. As Cabe and Wagman (2010) pointed out, perceptual learning with feedback on accuracy relative to an external scalar standard can affect both those parameters, typically moving the slope toward unity and the intercept toward zero, as well as increasing the variance accounted for $\left(R^{2}\right)$. A perceptual-learning manipulation would be worthwhile to attempt with a major axis localization task. Nevertheless, as Carello, et al. (1998) have suggested for scalar judgments in other dynamic touch tasks, the present measurements exhibit definite scaling (i.e., although not entirely accurate, in a highly novel task, they are scaled reasonably, relative to actual values).

These outcomes add another dimension to haptic distal spatial perception mediated by strings, in a dynamic touch mode. The data demonstrate that participants perceived string-mediated egocentric distance (i.e., between the exploring finger and the fixed position of the ellipse major axis), as suggested by the analysis in the Appendix, which showed that the geometry and physical-mechanical parameters available to the haptic system might support such perception.

\section{General discussion}

The results reported here are initial demonstrations of two types of haptic distal spatial perception mediated by strings, guided and constrained by ellipse geometry. Experiments $1 \mathrm{~A}$ and $1 \mathrm{~B}$ showed that perceivers could reliably differentiate and scale ellipse IFDs when their exploring forefingers traced paths limited by taut fixed-length strings attached to the foci. Experiment 2 showed that perceivers could reliably differentiate and scale the distal location of the ellipse major axes under similar exploratory conditions. The analyses in the Appendix proposed the possibility of such perceptual performance; the data reported document that, in fact, perceivers readily made the judgments hypothesized. Regression analyses using stimulus array variables suggested that the variables identified in the Appendix probably guided the estimates called for.

The derivations shown in the Appendix imply that the available information for IFD and major axis location involves three factors: (a) distance traveled by the finger along the ellipse perimeter, (b) the angle between string segments, and (c) changes in that angle. These variables are concomitantly present, because ellipse geometry constrained their values. Use of such variables is plausible, in at least general terms; the literature supports the availability of such factors to a potential perceiver. Alternatively, some research suggests that force relationships and changes in those (as examined in Cabe, 2011, and Cabe \& Hofman, 2012) might inform judgments. However, force relationships, while necessarily present, do not imply information 
for IFD or major axis localization under the conditions tested, because force magnitudes and direction at varying finger positions are not unique relative to either IFD or the major axis (i.e., many different force vectors could hold the string taut).

Below, I review literature related to haptic variables potentially available to perceivers in the tasks used in the present studies. In particular, these studies attest to a human capability to (a) estimate extent of movement guided by external objects (e.g., as the taut string was in the present studies), (b) judge hand-arm position as those effectors move, and (c) scale sizes of angles judged haptically (e.g., as the intersection of the two string segments at the fingertip presented in the present work).

\section{Haptic judgments of extent}

One parameter included in the analysis that led to Experiments $1 \mathrm{~A}$ and $1 \mathrm{~B}$ is the distance the finger traverses along the ellipse perimeter.

\section{Curved extents}

In one prominent example, Heller et al. (2008, Experiment 2) found that early and late blind, very low vision, and blindfolded sighted perceivers reasonably estimated distance along curved trajectories, as compared with straight lines of the same length. Estimates varied closely with actual length $(r>.97$, calculated from mean data presented in the article) for all sets of stimuli. Estimates were somewhat shorter than actual lengths, although estimates did not differ by line type or by visual status. Those results are clear evidence that perceivers can judge concave arc lengths, as proposed in the present experiments, with reasonable accuracy.

\section{Linear extents}

Other researchers have shown that perceivers can estimate linear distances reasonably well, using active or passive touch. One can also consider these as position changes guided by tactile stimuli.

In one useful example, Hollins and Goble (1988) studied perceivers' abilities to judge the lengths of straight metal rods, explored with a fingertip. They found excellent linear fits between actual lengths and magnitude estimations of length; power law exponents closely approximated 1.00, for both haptic and visual estimations. In a second experiment, Hollins and Goble instructed participants to move their fingers at ordinally different speeds over differing distances. While speeds actually produced varied both within and between participants, faster speeds generally were associated with shorter length estimates. Hollins and Goble derived a mathematical expression incorporating both actual length and exploratory speed and showed that their data fit that expression quite closely. Hollins and Goble also proposed credible physiological mechanisms that might underlie the effects reported. They emphasized that a given individual quite likely executes arm movements at a roughly constant exploration speed, leading to relatively well-scaled linear extent judgments. Their proposal invokes elements of both movement duration and limb position, elements clearly in play in the experiments reported in the present article.

Relatedly, but on a larger scale, Philbeck, Woods, Contra, and Zdenkova (2010) recently reported that their participants could readily match distances walked to lengths of rope pulled through the hands. That outcome, relative to the present experiments, suggests that humans can judge lengths of cordage moving across the hand or fingers reasonably well.

To summarize, the literature clearly indicates that human perceivers can readily judge movement extents along both linear and curved paths. As Hollins and Goble (1988) pointed out, there is a reasonable physiological basis for such judgments. Consequently, the use of such extent information in making string-mediated distal haptic perceptual judgments appears quite feasible.

\section{Sensitivity to hand-arm position}

The analysis in the Appendix also invokes sensitivity to changing hand-arm position as the finger moves along the elliptical path defined by the taut string. Drewing and colleagues (Drewing \& Ernst, 2006; Drewing \& Kaim, 2009; Drewing, Wiecki, \& Ernst, 2008; Kaim \& Drewing, 2010) have documented the utility of hand-arm position information along a curved path as a basis for haptic discrimination of (typically, Gaussian) curves. A number of other studies examining haptic curvature perception (e.g., Kappers, Koenderink, \& Lichtenegger, 1994) have implicated finger-hand-arm position information as a basis for discrimination of curvature differences. Relevant to Experiment 2 here, Pont, Kappers, and Koenderink (1999) tested base-topeak heights as a possible source of information for curvature discrimination. They found that thresholds for curved stimuli of constant radius were $0.5-1 \mathrm{~mm}$. That result suggests relatively precise sensitivity to, in effect, the derivative of the curve (i.e., in Cartesian coordinates, the rate of change in $y$ with respect to the rate of change in $x$, where $x$ and $y$ are related by the equation of the curve).

Heller et al. (2010), however, pointed out that haptically tracing curved paths can evoke illusory percepts of curve properties. In particular, Heller et al. (2010) had participants judge curve heights versus widths, when heights and widths were objectively equal, conditions that can be instantiated with ellipses or parabolas. Heller et al. (2010) found that 
height was generally overestimated relative to width, although the magnitude of the illusion depended on the manner in which participants explored the curve (e.g., with a single finger vs. bimanually or with the figure centered on the body midline vs. to one side).

These studies document human perceptual sensitivity to hand-arm position changes, especially as effectors are moved along curved trajectories. Thus, the element of hand-arm position changes, proposed in the Appendix as potentially informative for IFD and axis localization, is eminently plausible.

\section{Haptic judgments of angles}

The proposal underlying the present experiments is that participants can use the angle between string segments (or its supplement, the wrap angle), which changes as the finger explores the ellipse perimeter, as a basis for judging both IFD and major axis localization. While sensitivity to movement extent along curved paths has been studied fairly extensively, the literature on haptic angle judgments is both more limited and less clear.

Appelle (1971) investigated perceiver ability to match angles instantiated by two intersecting arms (wooden strips mounted on a backing panel) explored haptically. Using standard angles of $30^{\circ}-150^{\circ}$, he found mean differences between actual and estimated angles of about $6^{\circ}$. Voisin, Benoit, and Chapman (2001) found an average error of about $5^{\circ}$, using a $13^{\circ}$ range of angles versus a $90^{\circ}$ standard. Interestingly, Voisin et al. oriented the stimulus angles in a vertical plane, and participants explored them with outstretched arm-hand-forefinger, much as was done in the present experiments.

This literature lends some support to the contention laid out in the Appendix that change in string angle at the finger, as a function of movement of the string over the finger, could provide information for estimates of IFD, as in Experiments 1A and 1B. Similarly, change in wrap angle at the finger as a function of change in (vertical) finger position could provide information for major axis localization judgments, as in Experiment 2. The apparently relatively large threshold values for angle differentiation may be a source of variability in the IFD and major axis localization estimates reported here.

\section{Alternative explanations}

Multiple authors have offered explanations for haptic spatial property perception. Those fall into two broad categories: (a) force based and (b) attitude based. Both might speak to the results obtained in the present experiments and, therefore, are reviewed here as alternatives to the mechanisms proposed above.

\section{Force-based explanations}

Drewing and colleagues (Drewing \& Ernst, 2006; Drewing \& Kaim, 2009; Drewing et al., 2008; Kaim \& Drewing, 2010) pointed out that some haptic spatial perceptual effects (i.e., surface curvature judgments) apparently depend on an amalgam of information from changing finger position and force change, both available as the finger moves across a curved surface. Robles-de-la-Torre and Hayward (2001) demonstrated that when force and geometry (essentially, finger position) conflict, force relationships take priority in categorizing curved versus flat surfaces. Using a forcefeedback device, the latter authors imposed congruent or incongruent force and position conditions. When the forcefeedback information was incongruent with position information, force information dominated judgments.

Easton and Falzett (1978) found that finger pressure profiles tracked object curvature when participants moved their forefingers along a metal rod bent into a circular arc $37 \mathrm{~cm}$ in extent. The arc was attached to a pressure transducer, such that a continuous record of finger pressure was obtained during exploration. Song, Flanders, and Soechting (2004), however, concluded that their participants tended to produce a nearly constant normal force, whether finger movement was along an inwardly curved (convex toward the participant), outwardly curved, or straight path. In the experiment of Song et al., compliance (resistive force as a function of position) varied. It appears, therefore, that the issue of force information for curvature is still open and that a definitive answer depends on the particulars of the experimental situation.

Easton and Falzett (1978) are clear that they assume a constant finger pressure, normal to the curve, as do other investigators who have focused on force relationships in curvature judgments. However, Nakazawa, Ikeura, and Inooka (2000) found complex patterns of strain responses in fingertips to which shearing forces were applied in varying directions. Those authors also point out that much of the past research has given scant attention to tangential forces on fingertips.

While force changes as the finger traces a curve may inform perception of curvature, it is doubtful that such force change information can inform the ellipse IFD or major axis localization judgments examined in the present experiments, although force changes may provide information about the shape of the finger's trajectory. Given that the finger's trajectory is an ellipse, knowledge about its perimeter could imply other properties of the ellipse (although, see Heller et al., 2010). However, even that perspective must depend on assumptions about the nature of the force changes and their regularity. Because the individual participant controls his or her own movement, the particulars of applied force cannot be guaranteed in practice. In the present experiments, under 
the restriction that tension be maintained in both string segments between the finger and attachment points, the participant could generate an infinite number of force vectors, differing in magnitude and direction, so long as the vector is not coincident with either of the string segments. Changes in force vectors (magnitude and direction) are known to occur as individuals trace a curve (Easton \& Falzett, 1978), but with marked individual differences in such changes. Furthermore, there is clear evidence that fingertip tissues experience nonnormal forces (shearing), to which the haptic system is sensitive (e.g., Nakazawa et al., 2000). Hence, with respect to the experiments reported here, it appears more parsimonious to consider definable explanatory variables (e.g., wrap angle and egocentric finger position) that physically-mathematically relate to the outcome measures in a determinate fashion, rather than force magnitude and force changes that are indefinable and indeterminate.

\section{Attitude-based explanations}

Pont et al. (1999) defined attitude as the local slope of a curved surface, the angle between gravitational horizontal and the tangent to the curve at the point at which it is touched. Pont et al. presented data suggesting that their participants used attitude and attitude change to inform haptic spatial perception (curvature judgments), as the finger traced convex, concave, and straight trajectories. Supposing that the curve can be identified as an ellipse (perhaps in the sense of Pascal's well-known theorem that five co-planar, noncollinear points define a conic), it is possible, in principle, that attitude changes could lead to IFD and major axis localization, much as force changes might. That mathematics is not obvious, however, and (again) it seems more parsimonious to propose that elements identified in the Appendix here seem more likely to have been used.

Strings as haptic mediators

Strings, as shown here and in other research, can mediate at least some aspects of haptic distal spatial perception. Flexible, light-weight strings differ from other manipulanda (e.g., a blind person's cane) in that their physical properties (e.g., mass, linear dimensions) are negligible (see the extended discussion in Cabe, 2011). Rather, the important physical-mechanical elements that allow such mediation are string's resistance to tension and flexibility, both clearly present in the apparatus used for the present studies.

The mediating function of strings is analogous - with obvious limitations - to that of light rays for vision and sound waves for hearing. Gibson (1966) pointed out that the properties of light per se are not important for vision, nor are the properties of sound per se important for audition. Instead, it is the invariant structure of time-evolving (transforming) light patterns across the optic array and the invariant structure of time-evolving (transforming) sound patterns across acoustic arrays that are information for distal environmental properties. Thus, light in optic arrays and sound in acoustic arrays are media that contain information for visual and auditory perception. Similarly, the claim supported by the present work, as well as that of Cabe (2011) and Cabe and Hofman (2012), is that (transforming) geometric angular relations among string segments, and (transforming) force relationships attendant to such geometries, mediate information for haptic perception of environmental features distal to the perceiver. Those relationships appear to be invariant over changing values of angle, force, and other transforming factors.

Cabe (2011; Cabe \& Hofman, 2012) cited an anecdote from Molyneaux's well-known "Letter on the Blind" (Morgan, 1977). Molyneaux described Saunderson (the blind Cambridge mathematician who succeeded Newton; Tattersall, 1992) as teaching optics using stretched strings to represent light rays. Kennedy (1974) pointed out that drawn lines can represent strands, as in a wire fence; similarly, lines can represent light rays (e.g., in ray tracing diagrams). Thus, optical geometric analysis is the foundation for visual perception (e.g., Sedgwick, 1986). Within practical limits (perhaps narrow), the geometry of taut strings mimics the geometry of light rays, and the angular relationships that occur both with light and with taut strings may provide similar information for distal spatial perception.

An additional pilot experiment related to those reported here examined a further important spatial variable: participants' ability to estimate the slant of the major axes of ellipses, relative to gravitationally defined horizontal, while moving their forefingers along the string-defined ellipse perimeter. Geometric analysis suggested the availability of information for such perception. However, correlations between actual slant and estimated slant for 5 of the 6 participants tested were nonsignificant $(r=.07-.21$; minimum $p=.11 ; n=56$ data points, 7 slant values $\times 8$ trial blocks). One participant nevertheless showed a relatively strong correlation, $r=.74, p<.0001$, hinting that available information might be used to make such judgments. Those tentative outcomes suggest that perceptual learning manipulations might allow participants to either discover or improve use of available information for slant.

The novelty of string-mediated haptic distal spatial perception warrants some passing comment. ${ }^{4}$ One might suggest, for example, that the tasks imposed in the present studies, as well as in past work, lack ecological validity (i.e., they have few or no analogs in everyday experience). While that may be generally true, a number of arguments justifying the present use of a novel experimental task can be offered. First, it serves as a

\footnotetext{
${ }^{4}$ This section repeats, in part, arguments also included in Cabe (2010, Footnote 2; 2011), and Cabe and Hofman (2012).
} 
test case for direct perception, per Gibson (1966). Gibson (1966) argued that all perception is determined by pickup of informative structure in stimulus arrays and, thus, that inputs to perceptual systems need not be "embellished, organized, structured, and repaired so as to yield adequate perception" (Michaels \& Carello, 1981, p. 8). Aurell (1984), however, has pointed out that direct perception and automatized, memorially supported perception are indistinguishable. Consequently, tasks largely beyond the perceiver's routine experience, yet resulting in successful perceptual performance, suggest support for the Gibsonian perspective. Conversely, if perception depends strongly on the kind of memorial processes that Aurell addresses, performance should be weak or not possible (see Cabe, 1976, for one application of this same logic).

Second, if one's aim is to discover latent perceptual capabilities, one perforce must use novel tasks. Haptic distal spatial perception is just such a latent capability; the data presented here, as well as in the few other investigations extant, demonstrate its existence. Cabe and Hofman (2012) described additional examples of the general theme of discovering latent capabilities. Finally, to directly address the issue of ecological validity, Cabe (2011) adduced several examples of string-mediated perception-action performance.

Open questions and directions for future research

The results of the experiments reported here document human string-mediated haptic capability to perceive size (separation of points) at a distance and distal localization of spatial features, relative to a moving observation point. Those results suggest a variety of elaborations, extensions, and expansions to be examined in future research, opening the door to a potentially rich research program. Some possibilities are briefly described below.

An immediate target for additional studies would be variations on the paradigm employed in the present work. A first step would be to parametrically examine IFD and string length, because the present experiments sampled only a relatively small range of those, in order to establish that the effect occurs over a broader range of conditions. Additionally, to directly test the proposition that string angle change informs IFD and localization judgments, participants might explore the ellipse perimeter using a manipulandum (cf. Cabe, 2011), to eliminate string angle information. Furthermore, it would be of interest to discover the degree to which direct estimates of string angle (more to the point, wrap angle, the degree of contact between the string and the finger surface) can be made, since relatively sparse data appear to exist on that general issue. Because changing finger-arm position is potentially also a source of information for those distal spatial features, specific manipulations of such exploration could be imposed (e.g., limiting the velocity, extent, and number of exploratory movements). Investigating the possible effects of response parameters (e.g., some active response vs. the verbal response employed in Experiment 1A) would be useful. Finally, because scaling in Experiments $1 \mathrm{~B}$ and 2 showed notable error, perceptual learning manipulations would be of interest, to see to what degree scaling might improve with feedback on judgment accuracy.

Three categories of information appear to be involved in string-mediated haptic distal spatial perception: (a) angle magnitudes between string segments and their changes with finger movement, (b) force vector magnitudes and directions and their changes with finger movement, and (c) finger position changes. Studies so far (Cabe, 2011; Cabe \& Hofman, 2012; the present experiments) have attempted to isolate various combinations of those variables. Cabe (2011) invoked all three, then (by use of manipulanda) attempted to remove string angle information. Cabe and Hofman removed string angle information by employing only a single string and a ring at the participant's finger; force vector and finger position changes remained.

The Appendix suggests that a combination of string angle change and finger position change could inform the estimates called for in the present experiments. Those changes, in the present work, were constrained by ellipse geometry. Yet perceivers might be able to make similar estimates, reasonably reliably and reasonably accurately, if finger movements were unconstrained by any particular finger trajectory geometry. Unlike the physical arrangement that yielded ellipse geometry, which aimed to minimize force-based information, unrestricted movements would likely invoke combinations of force, string angle, and finger position changes.

It would be interesting to find additional ways to vary the three main classes of informational variables proposed for IFD and major axis localization. For example, it might be possible to move the foci of the ellipse, while keeping the finger in one position; if so, the passive-active haptic exploration distinction cited in a variety of past studies (see Lederman \& Klatzky, 2009) might be examined.

\section{Appendix}

Geometric informational analysis of the haptic stimulus array, based on spatial properties of ellipses

The general position adopted here is that stimulus array information can be said to exist for some to-be-perceived environmental property if a physical-mathematical expression for the property in question can be derived in terms of potentially sensible array properties, also described in physical-mathematical terms. That position makes no special assertions about the nature of the relationship (e.g., an equation) between the environmental property and the stimulus array properties, only that such a relationship can be 
shown. If any such relationship exists, stimulus array information may be said to exist, no matter how simple or complicated the relationship turns out to be. Typically, given a mobile observer (or changing observation point), one expects those relationships to involve both momentary values of the relevant variables and changes in them (e.g., with respect to time or to other variables). Alternative expressions may exist for any given relationship between an environmental property and stimulus array variables. Such is the case with the conclusions reached below; the ones described have the advantage of being reasonably compact.

An ellipse can be defined as the locus of the apices of a set of triangles with a fixed base and a constant perimeter (acknowledging that such triangles become degenerate, a straight line, with the apex at either end of the ellipse major axis, or if the base is zero, yielding a circle). That definition can be applied to drawing an ellipse by passing a loop of string around two fixed pins and pulling the loop tight with a pencil tip. Its movement constrained within the loop, the pencil draws an ellipse. Ellipses differ as a function of two parameters, the distance between the fixed points and the triangles' constant perimeter.

The apical angles of these triangles are maximal when the apex is at either end of the ellipse minor axis and zero at the ends of the major axis. When the ratio of the length of the semi-minor axis to the distance from the center to either focus is 1.00 , the maximum angle is $90^{\circ}$. If that ratio is less than 1.00 , the maximum angle is greater than $90^{\circ}$; if the ratio is more than 1.00 , the maximum angle is less than $90^{\circ}$. As the ratio increases, the maximum angle decreases to a limit of zero (defining a circle). Note that the length of the semi-minor axis always must exceed the distance from the center to a focus, if the ellipse is not degenerate.

Availability of haptic information for ellipse interfocal distance

If, instead of a pencil, a moving finger holds the string taut, the finger's path describes an elliptical trajectory (Fig. 1). The angle between the string segments at the finger varies as the finger moves. If the string attachment points (the ellipse foci) are not known, no single string angle can give their positions; the triangle formed by the apex angle and the string segments is indeterminate. However, given two fixed hook positions and a fixed string length (greater than the distance between the hooks), any two finger positions (and thus, two angles between string segments) unambiguously locate the positions of the hooks: The two resulting triangles have a common base, the ellipse IFD. Stated in terms of progressive change, the differential of the angle as a function of a differential of finger movement (which can be construed in several ways) can yield the IFD.

Per Fig. 5, with the ellipse centered on Cartesian axes, one hook is located at $(\mathrm{C}, 0)$, the other is at $(-\mathrm{C}, 0)$, the two foci of

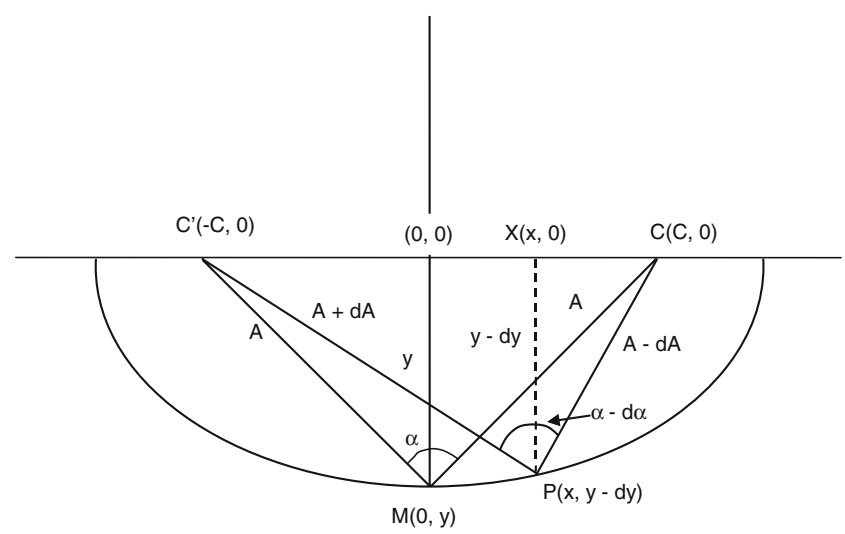

Fig. 5 The geometry of a generalized ellipse. For explanations of the symbols, refer to the Appendix

the ellipse. The string is of length $2 A$, with $A>C$. When the finger is directly below the Cartesian origin (point M in Fig. 5), the string segments are both of length $A$, forming angle $\alpha$ at the finger. If the finger moves some distance away from the end of the semi-minor axis, still holding the string taut, the string segments now are of length $A+d A$ and $A-d A$, where $d A$ is the change in the lengths of the two string segments and, because the string is of fixed length, also the portion of the string that has moved across the finger surface as the finger changes location along its elliptical track and, evidently, the distance the finger has moved along the perimeter of the ellipse.

With the finger centered below the axis origin (i.e., at the end of the ellipse semi-minor axis), by the law of cosines,

$\operatorname{COS}(\alpha)=\frac{A^{2}-2 C^{2}}{A^{2}}$

where $\alpha$ is the angle between the two string segments, $A$ is the length of each string segment between the finger and either of the foci, and $C$ is the distance from the origin to either of the foci (and $2 C$ therefore the IFD). Differentiating and rearranging yields

$4 C^{2}=-A^{3}[\operatorname{SIN}(\alpha)] \frac{d \alpha}{d A}$.

Solving Eq. 1 for $-\mathrm{A}$ (because the half-ellipse in question is below the major axis; see Fig. 5), substituting the resulting expression into Eq. 2, simplifying and solving for $C$ gives two solutions, $C=O$ (in which case the ellipse would be a circle) and

$C=\frac{\sqrt{2}[1-\operatorname{COS}(\alpha)]^{3 / 2}}{\operatorname{SIN}(\alpha) \frac{d \alpha}{d A}}$.

If $C$ is nonzero, $2 C$ is the ellipse IFD. Thus, $C$ (an environmental property) is specified by a relationship among array properties: angle $\alpha$ and the change in that angle as a function of the change in the string segment lengths (i.e., $d \alpha /$ $d A$ ). Note that the constraint that string length, $2 A$, is 
constant means that $d A$ must be added to one string segment and subtracted from the other.

Consequently, one can hypothesize that perceivers might be able to recover IFD if allowed to haptically explore the ellipse formed by a given string length and a given IFD. As the finger moves, the perceiver's haptic system has available to it the angle $\alpha$, the distance the finger has moved along the ellipse perimeter $(d A)$, and the resulting change in the angle $(d \alpha)$. The degree of string contact with the finger (termed wrap angle by Cabe, 2011) varies as the supplement of $\alpha$.

Interestingly, the relationship in Eq. 3 is independent of the actual length of the string; the variable $A$ does not appear at all. Rather, it is only the angle itself and the change in the angle (i.e., $d \alpha$ ) as a function of change in position along the string (i.e., $d A$ ) that matters. IFD, per Eq. 3, therefore is specified invariantly with respect to string length. Thus, the relationship in Eq. 3 generalizes to any nondegenerate ellipse. String length does, of course, affect the sizes of the angles that the finger could explore, with the maximum angle varying inversely with string length for a given IFD, so string length might or might not be an influential independent variable in empirical tests of IFD judgments.

\section{Availability of haptic information for ellipse major axis localization}

With the finger placed at the end of the minor axis, the tangent of the angle between the string segments at the finger is

$\operatorname{TAN}(\alpha)=\frac{2 C y}{y^{2}-C^{2}}$,

where $\alpha$ is the angle between the string segments, $C$ is the distance from the ellipse center to one of the foci, and $y$ is the length of the semi-minor axis. We would like to find an expression for $y$.

Solving for $\mathrm{C}$ and taking the positive root gives

$C=y\left[\frac{\operatorname{SIN}(\alpha)}{\operatorname{COS}(\alpha)+1}\right]$.

Differentiating Eq. 4 and rearranging yields

$\operatorname{SEC}(\alpha)^{2} d \alpha=\left[\frac{2 C\left(C^{2}+y^{2}\right)}{\left(C^{2}+y^{2}\right)^{2}}\right] d y$.

Substituting the expression for $C$ from Eq. 5 into Eq. 6 and solving for $y$ yields

$y=[-\operatorname{SIN}(\alpha)] \frac{d y}{d \alpha}$.

Note that both the angle between the string segments and the perpendicular distance from the finger to the major axis decrease as the finger moves from the end of the semi-minor axis.
Equation 7 indicates that, if perceivers can pick up the angle between the strings at the fingertip, as well as the change in the angle and the change in the finger's vertical distance as the finger moves (all of which are in principle available to the haptic system), then they have access to haptic information for the location of the ellipse major axis. The relationship in Eq. 7, moreover, is invariant with respect to both string length and IFD, so the relationship holds generally over all ellipses. Again, however, the range of angles available depends on string length for a given IFD, so string angle might or might not affect empirical judgments of major axis localization.

\section{References}

Aleci, C., Piana, G., \& Anselmino, F. (2010). Evaluation of spatial anisotropy by curvature analysis of elliptical targets. The Open Ophthalmology Journal, 4, 15-21.

Amazeen, E. L. (1999). Perceptual independence of size and weight by dynamic touch. Journal of Experimental Psychology: Human Perception and Performance, 25, 102-119.

Appelle, S. (1971). Visual and haptic angle perception in the matching task. American Journal of Psychology, 84, 487-499.

Aurell, C. G. (1984). Note on Gibson's direct visual perception. Perceptual and Motor Skills, 58, 540-542.

Burton, G. (1993). Non-neural extensions of haptic sensitivity. Ecological Psychology, 5, 105-124.

Burton, G., Turvey, M. T., \& Solomon, H. Y. (1990). Can shape be perceived by dynamic touch? Perception \& Psychophysics, 48, 477-487.

Cabe, P. A. (1976). Transfer of discrimination from solid objects to pictures by pigeons: A test of theoretical models of picture perception. Perception and Psychophysics, 19, 545-550.

Cabe, P. A. (2010). Sufficiency of longitudinal moment of inertia for haptic cylinder length judgments. Journal of Experimental Psychology: Human Perception and Performance, 36, 373-394.

Cabe, P. A. (2011). Haptic distal spatial perception mediated by strings: Haptic "looming. Journal of Experimental Psychology: Human Perception and Performance, 37, 1492-1511.

Cabe, P. A., \& Hofman, L. L. (2012). Haptic distal spatial perception mediated by strings: Point of closest approach and bypass distance. Journal of Experimental Psychology: Human Perception and Performance, 38, 1328-1340.

Cabe, P. A., \& Wagman, J. B. (2010). Characterizing perceptual learning using regression statistics. American Journal of Psychology, 123, 253-267.

Cabe, P. A., Wright, C. D., \& Wright, M. A. (2003). Descartes' blind man revisited: Kinesthetic triangulation of distance using handheld rods. American Journal of Psychology, 116, 73-100.

Carello, C., Fitzpatrick, P., Flascher, I., \& Turvey, M. T. (1998). Inertial eigenvalues, rod density, and rod diameter in length perception by dynamic touch. Perception \& Psychophysics, 660, 89-100.

Carello, C., \& Turvey, M. T. (2004). Physics and psychology of the muscle sense. Current Directions in Psychological Science, 13, $25-28$.

Chan, T.-C., \& Turvey, M. T. (1991). Perceiving the vertical distances of surfaces by means of a hand-held probe. Journal of Experimental Psychology: Human Perception and Performance, 17, 347-358. doi:10.1037/0096-1523.17.2.347

Cutting, J. E. (1986). Perception with an eye for motion. Cambridge, MA: MIT Press. 
Dresp, B., Silvestri, C., \& Motro, R. (2007). Which geometric model for the curvature of 2-D shape contours? Spatial Vision, 20, 219-264.

Drewing, K., \& Ernst, M. O. (2006). Integration of force and position cues for shape perception through active touch. Brain Research, 1078, 92-100.

Drewing, K., \& Kaim, L. (2009). Haptic shape perception from force and position signals varies with exploratory movement direction and the exploring finger. Attention, Perception, \& Psychophysics, 71, 1174-1184. doi:10.3758/APP.71.5.1174

Drewing, K., Wiecki, T. V., \& Ernst, M. O. (2008). Material properties determine how force and position signals combine in haptic shape perception. Acta Psychologica, 128, 264-273.

Easton, R. D., \& Falzett, M. (1978). Finger pressure during tracking of curved contours: Implications for a visual dominance phenomenon. Perception \& Psychophysics, 24, 145-153.

Garrett, S., Barac-Cikoja, D., Carello, C., \& Turvey, M. T. (1996). A parallel between visual and haptic perception of size at a distance. Ecological Psychology, 8, 25-42.

Gibson, J. J. (1962). Observations on active touch. Psychological Review, 69, 477-491.

Gibson, J. J. (1966). The senses considered as perceptual systems. Boston: Houghton Mifflin.

Gibson, J. J. (1979). The ecological approach to visual perception. Boston: Houghton Mifflin.

Hayward, V. (2008). Haptic shape cues, invariants, priors, and interface design. In M. Grunwald (Ed.), Human haptic perception: Basics and applications (pp. 381-292). Basel: Birkhäuser Verlag.

Heller, M. A., Kappers, A. M. L., McCarthy, M., Clark, A., Riddle, T., Fulkerson, E., \& Russler, K. (2008). The effects of curvature on haptic judgments of extent in sighted and blind people. Perception, 37, 816-840. doi:10.1068/p5497

Heller, M. A., Walk, A. D. M., Schnarr, R., Kibble, S., Litwiller, B., \& Ambuehl, C. (2010). Attenuating the haptic horizontal-vertical curvature illusion. Attention, Perception, \& Psychophysics, 72, $1626-1641$.

Hollins, M., \& Goble, A. K. (1988). Perception of length of voluntary movements. Somatosensory Research, 5, 335-348.

Jacobs, D. M., \& Michaels, C. F. (2007). Direct learning. Ecological Psychology, 19, 321-349.

Kaim, L., \& Drewing, K. (2010). Exploratory pressure influences haptic shape perception via force signals. Attention, Perception, \& Psychophysics, 72, 823-838. doi:10.3758/APP.72.3.823

Kaiser, M. K., \& Mowafy, L. (1993). Optical specification of time-topassage: Observers' sensitivity to global tau. Journal of Experimental Psychology: Human Perception and Performance, 19, $1028-1040$

Kappers, A. M. L., Koenderink, J. J., \& Lichtenegger, I. (1994). Haptic identification of curved surfaces. Perception \& Psychophysics, 56, 53-61.

Kennedy, J. M. (1974). A psychology of picture perception. San Francisco: Jossey-Bass.

Kinsella-Shaw, J. M., \& Turvey, M. T. (1992). Haptic perception of object distance in a single-strand vibratory web. Perception \& Psychophysics, 52, 625-638.

Lederman, S. J., \& Klatzky, R. L. (1993). Extracting object properties through haptic exploration. Acta Psychologica, 84, 29-40.

Lederman, S. J., \& Klatzky, R. L. (2009). Haptic perception: A tutorial. Attention, Perception, \& Psychophysics, 71, 1439-1459. doi:10.3758/APP.71.7.1439
Lee, D. N. (1976). A theory of visual control of braking based on information about time-to-collision. Perception, 5, 437-459.

Lee, D. N. (2009). Lee's 1976 paper. Perception, 38, 837-858. doi:10.1068/ldmk-lee

Michaels, C. F., \& Carello, C. (1981). Direct perception. Englewood Cliffs, NJ: Prentice-Hall.

Morgan, M. J. (1977). Molyneux's question: Vision, touch and the philosophy of perception. New York: Cambridge University Press.

Nakazawa, N., Ikeura, R., \& Inooka, H. (2000). Characteristics of human fingertips in the shearing direction. Biological Cybernetics, 82, 207 214.

Philbeck, J. W., Woods, A. J., Contra, C., \& Zdenkova, P. (2010). A comparison of blindpulling and blindwalking as measures of perceived absolute distance. Behavior Research Methods, 42, 148-160. doi:10.3758/BRM.42.1.148

Pont, S. C., Kappers, A. M. L., \& Koenderink, J. J. (1999). Similar mechanisms underlie curvature comparison by static and dynamic touch. Perception \& Psychophysics, 61, 874894.

Robles-de-la-Torre, G., \& Hayward, V. (2001). Force can overcome object geometry in the perception of shape through active touch. Nature, 412, 445-448.

Sedgwick, H. A. (1973). The visible horizon: A potential source of visual information for the perception of size and distance. Dissertation Abstracts International, 34, 1301-1302B. UMI No. 7322530 .

Sedgwick, H. A. (1986). Space perception. In K. R. Boff, L. Kaufman, \& J. P. Thomas (Eds.), Handbook of perception and human performance, Vol. 1: Sensory processes and perception (pp. 211-21-57). New York: Wiley.

Song, W., Flanders, M., \& Soechting, J. F. (2004). Effect of compliance on haptic perception of curvature. Somatosensory \& Motor Research, 21, 177-182.

Tattersall, J. J. (1992). Nicholas Saunderson: The blind Lucasian professor. Historia Mathematica, 19, 356-370.

Turvey, M. T. (1996). Dynamic touch. American Psychologist, 51, $1134-1152$.

Turvey, M. T., \& Carello, C. (1995). Dynamic touch. In W. Epstein \& S. Rogers (Eds.), Perception of space and motion (pp. 401-490). San Diego: Academic.

Turvey, M. T., Solomon, H. Y., \& Burton, G. (1989). An ecological analysis of knowing by wielding. Journal of the Experimental Analysis of Behavior, 52, 387-407.

Van der Horst, B. J., \& Kappers, A. M. L. (2008). Using curvature information in haptic shape perception of 3D objects. Experimental Brain Research, 190, 361-367. doi:10.1007/s00221-008-1478-6

Voisin, J., Benoit, G., \& Chapman, C. E. (2001). Haptic discrimination of object shape in humans: Two-dimensional angle discrimination. Experimental Brain Research, 145, 239-250. doi:10.1007/ s00221-002-1117-6

Von Fieandt, K. (1966). The world of perception. Homewood, IL: Dorsey.

Wightman, F. L., \& Jenison, R. (1995). Auditory spatial layout. In W. Epstein \& S. Rogers (Eds.), Perception of space and motion (pp. 365-400). San Diego: Academic.

Withagen, R., \& Caljouw, S. R. (2011). Aging affects attunement in perceiving length by dynamic touch. Attention, Perception, \& Psychophysics, 73, 1216-1226. doi:10.3758/s13414-011-0092-z 